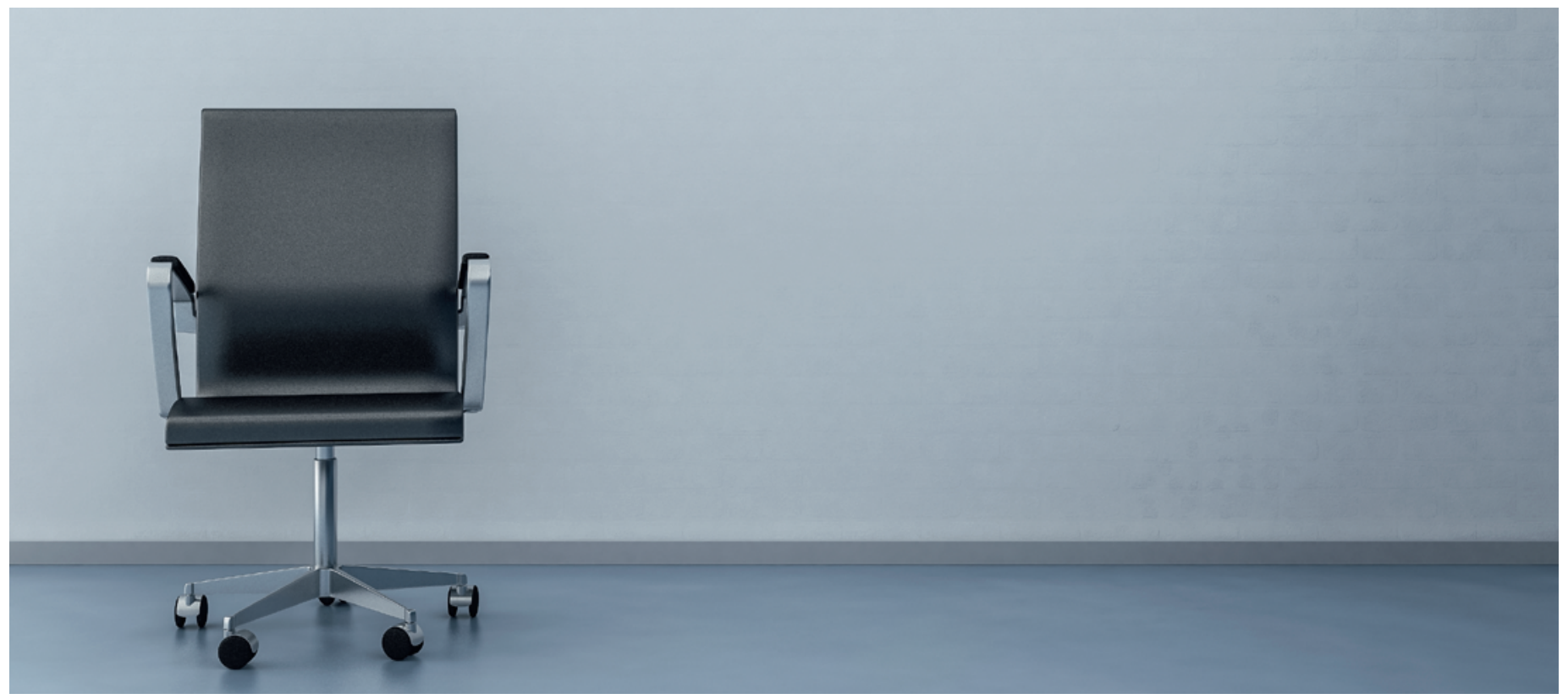

\title{
Warum Ärzte den Chefarztposten aufgeben
}

\section{Mirjam Thannera ${ }^{\text {, Kristina Milojkovic }}{ }^{b}$, René Hornung ${ }^{c}$}

${ }^{a}$ Dr. rer. pol., Betriebswirtschafterin der Frauenklinik, Kantonsspital St. Gallen; b Projektmitarbeiterin der Frauenklinik, Kantonsspital St. Gallen; c Prof. Dr. med., MBA, Chefarzt der Frauenklinik, Kantonsspital St. Gallen, Mitglied FMH

\section{Um mögliche Ursachen für die Aufgabe einer ärztlichen Führungsposition zu ermitteln, führten die Autoren Interviews mit ehemaligen Chefärztinnen und Chefärzten. Den Anstoss zur Untersuchung gab der subjektive Eindruck, dass in jüngster Zeit zahlreiche ärztliche Führungspersonen vor Erreichen des regulären Rentenalters die Chefarztposition aufgegeben hatten.}

\section{Pourquoi les médecins renoncent-ils au poste} de médecin-chef?

Le poste de médecin-chef est globalement considéré comme l'objectif de carrière par excellence dans l'activité clinique. Lorsque son titulaire renonce à cette fonction de direction hospitalière avant d'avoir atteint l'âge de la retraite, cela soulève des interrogations sur les motifs de son choix. Afin de comprendre ceux-ci à la lumière de l'idéal professionnel, 14 ancien(ne)s médecins-chef(fe)s en gynécologie et obstétrique de Suisse ont été interrogé(e)s. II en ressort que le médecin-chef idéal exerce dans un domaine médicalement exigeant, attache une grande importance au travail en équipe et à l'encouragement de la relève et est présent et joignable quasiment en permanence. La renonciation au poste de direction médicale était souvent due, outre la forte charge administrative et les droits de regard insuffisants en matière de décisions liées à l'activité clinique, à une mauvaise culture de l'organisation et à des incitations à la performance inappropriées.
«Was ist denn los? Wir haben bald keine Chefärzte mehr! Und wenn es so weiter geht, sind alle weg." (Aussage eines Interviewpartners)

Die berufliche Position des Chefarztes gilt gemeinhin als eigentliches Karriereziel klinischer Tätigkeit. Erfolgt die Aufgabe dieser ärztlichen Führungsposition im Spital vor Eintritt des Rentenalters, werden Fragen zu den Gründen aufgeworfen. Um diese vor dem Hintergrund des beruflichen Idealbildes nachvollziehen zu können, wurden Interviews mit 14 ehemaligen Chefärztinnen und Chefärzten des Fachgebiets Gynäkologie und Geburtshilfe in der Schweiz geführt und mittels qualitativer Inhaltsanalyse ausgewertet. Da die relevante Grundgesamtheit überschaubar war, soll im Folgenden auf eine detaillierte sozioökonomische Be- 
Tabelle 1: Ergebnisse der standardisierten Befragung ( $n=13$, fehlende Antworten $n=1)$.

\begin{tabular}{|c|c|}
\hline $\begin{array}{l}\text { In der Literaturrecherche und der aktuellen Diskussion } \\
\text { in den Medien sind wir vorab auf einige mögliche Gründe } \\
\text { gestossen, die dazu führen können, dass ein Chefarzt seine } \\
\text { Position aufgibt. Inwieweit können Sie diesen zustimmen? }\end{array}$ & $\begin{array}{l}\text { Anzahl der Befragten, } \\
\text { welche dem genannten } \\
\text { Grund "völlig" oder } \\
\text { "eher» zustimmten }\end{array}$ \\
\hline $\begin{array}{l}\text { Steigende Belastung durch administrative Tätigkeiten } \\
\text { im Vergleich zu den klinischen Tätigkeiten im Berufsalltag }\end{array}$ & 11 von 13 \\
\hline Keine angemessene Verortung in der Klinikhierarchie & 8 von 13 \\
\hline $\begin{array}{l}\text { Verschlechterung der Betriebskultur, z.B. fehlende Wert- } \\
\text { schätzung, schlechte Stimmung }\end{array}$ & 7 von 13 \\
\hline Unangemessene Leistungsanreize, z.B. Fallzahlen & 7 von 13 \\
\hline $\begin{array}{l}\text { Zu hohe Arbeitsbelastung im Vergleich zur Freizeit } \\
\text { (Work-Life-Balance) }\end{array}$ & 6 von 13 \\
\hline Mangelnde Vereinbarkeit mit der ärztlichen Berufsethik & 6 von 13 \\
\hline Ökonomisierung der ärztlichen Tätigkeit & 5 von 13 \\
\hline $\begin{array}{l}\text { Zunehmende Kontrolle der ärztlichen Tätigkeit, } \\
\text { zunehmendes Misstrauen }\end{array}$ & 5 von 13 \\
\hline Gehaltseinbussen & 5 von 13 \\
\hline Steigender juristischer Rechtfertigungsdruck & 5 von 13 \\
\hline Angst vor "Burnout» & 4 von 13 \\
\hline Zu hohe Verantwortung & 2 von 13 \\
\hline $\begin{array}{l}\text { Steigende Exposition als Chefarzt in den Medien, } \\
\text { z.B. bei Behandlungsfehlern }\end{array}$ & 1 von 13 \\
\hline Einbussen an Sozialprestige & 1 von 13 \\
\hline
\end{tabular}

schreibung der Gesprächspartner verzichtet werden, um deren Anonymität zu wahren.

Folgende Fragestellungen flossen in die Gespräche ein:

- Was waren die Ursachen für Ihre Entscheidung, die Stellung als Chefärztin / als Chefarzt aufzugeben?

- Welches Idealbild haben Sie von «einer guten Chefärztin / einem guten Chefarzt»?

Die Interviews ergaben ein breites Spektrum an Gründen, die zur Aufgabe der ärztlichen Führungsposition geführt hatten. Neben einer zu hohen Belastung durch administrative Tätigkeiten und zu geringen Mitspracherechten bei klinikrelevanten Entscheidungen spielten auch verschiedene Aspekte einer schlechten Organisationskultur eine Rolle.

Um Anhaltspunkte hinsichtlich der Priorität möglicher Gründe zu erhalten, wurden die Gesprächspartner unmittelbar im Anschluss an die Leitfadeninterviews gebeten, anhand eines standardisierten Fragebogens das Ausmass ihrer Zustimmung auf einer ordinalen vierstufigen Antwortskala auszudrücken (von «trifft gar nicht zu» über "trifft eher nicht zu» und "trifft eher zu» bis «trifft völlig zu»). Tabelle 1 zeigt die Anzahl der Befragten, welche dem jeweils genannten Grund «völlig» oder «eher» zustimmten, im Überblick.

\section{Der ideale Chefarzt: immer präsent, immer erreichbar}

Die Gesprächspartner zeichneten in den Interviews ein Idealbild von Chefärztinnen und Chefärzten, wel- che eine medizinisch anspruchsvolle Tätigkeit ausführen, für die Teamarbeit und Nachwuchsförderung grosse Bedeutung besitzen und deren persönliches Engagement auch durch hohe zeitliche Präsenz im Spital und schnelle Erreichbarkeit spürbar ist. Extrem lange Arbeitszeiten scheinen damit nicht nur zur beruflichen Realität von Chefärztinnen und Chefärzten, sondern auch zu deren Idealbild zu gehören. Dies ist relevant, da ein übermächtiges Idealbild auch ein Hemmnis für die Durchsetzung und Verbreitung von Jobsharing-Stellen im ärztlichen Führungsbereich sein kann. Solange Teilzeit-Chefärztinnen und TeilzeitChefärzte nicht mit dem Idealbild vereinbar sind, bleibt einer ärztlichen Führungsperson, die mehr Freizeit mit Familie oder Freunden verbringen will, wohl tatsächlich nur der Ausweg, dieses Amt aufzugeben. Im vorgestellten Projekt liessen sich aus betriebswirtschaftlicher Sicht Anhaltspunkte dafür finden, wie die Bindung von ärztlichen Führungspersonen an das Spital gelingen kann: Anstrengungen zur Verbesserung der Organisationskultur, mehr Mitspracherechte bei klinikrelevanten Entscheidungen und administrative Entlastung sind keine überraschenden Erfolgsfaktoren. Die Herausforderung liegt vielmehr darin, diese nicht nur zur Kenntnis zu nehmen, sondern auch im klinischen Alltag zu leben und konkret umzusetzen. Die Ergebnisse sprechen zudem für einen sorgsamen und reflektierten Einsatz von Leistungsanreizen. Weiterführende Studien zur organisationsbezogenen Versorgungsforschung wären hier wünschenswert. Während Aufgaben zur Verbesserung der Organisationskultur hauptsächlich interprofessionell zu lösen sind, erfordert die Fragestellung, inwieweit Teilzeitstellen oder gezielte berufliche Auszeiten für eine bessere Work-Life-Balance zum Idealbild «einer guten Chefärztin / eines guten Chefarztes» passen, in erster Linie eine Diskussion unter Ärztinnen und Ärzten. Es ist zu vermuten, dass nur das klare berufspolitische Bekenntnis dazu zum notwendigen Rückenwind verhilft, diese Vorstellungen auch zu verwirklichen.

\section{Anmerkungen}

Methodik und Ergebnisse der qualitativen Interviews wurden ausführlich dargestellt und diskutiert in der Publikation: Thanner M, Milojkovic K, Hornung R, Berufliches Ideal und Gründe zur Aufgabe einer Führungsposition, in: das Krankenhaus, Jg. 108, Heft 7 (2016): S. 584-8.

Bildnachweis

(C) Hofpils | Dreamstime.com 